\title{
ESTUDIO DE DETECCIÓN DEL RIESGO DE DIABETES EN ATENCIÓN PRIMARIA SEGÚN CUESTIONARIO FINDRISC EN EL MUNICIPIO DE GRAL. PUEYRREDÓN (ESTUDIO DR. DIAP)
}

\author{
DIABETES RISK DETECTION STUDY IN PRIMARY CARE ACCORDING \\ TO FINDRISC QUESTIONNAIRE IN THE MUNICIPALITY OF \\ GRAL.PUEYRREDÓN (DR. DIAP STUDY)
}

\begin{abstract}
Segundo Guzmán Rodríguez¹, María Cristina Faingold², Raúl Suárez³, Sofía Guzmán Rodríguez ${ }^{4,6,7}$, Mariel López Priori ${ }^{6,7}$, Jorge Martínez Arca ${ }^{5}$, Alejandra Lalli ${ }^{6,7}$, Anastasia Bonanno ${ }^{6,7}$, Griselda Bozzone ${ }^{6,7}$, Gabriela Pagani $^{6,7}$, Esteban Dottavio ${ }^{7}$, Laura Orosco ${ }^{7,8}$, Guillermo Alzueta $^{7,9}$, Adriana Scandurra ${ }^{5}$, Lucía Isabel Passoni $^{8}$, Carlos Ciccioli ${ }^{7}$, Leandro Leoni ${ }^{6,7}$, Débora Zantleifer ${ }^{6,7}$, María Laura Ruiz ${ }^{6,7}$, Manuela Rodríguez ${ }^{6,7}$
\end{abstract}

\section{RESUMEN}

Objetivos: conocer la magnitud del riesgo de padecer diabetes mellitus 2 (DM2) en la población del Municipio de Gral. Pueyrredón que concurre a los Centros Asistenciales de Atención Primaria.

Materiales y métodos: estudio observacional para determinar el riesgo de padecer DM2 mediante una entrevista donde se indagaron sobre las ocho preguntas del cuestionario FINDRISC.

Resultados: la muestra del estudio estuvo constituida por 2.784 pacientes, el 54\% conformada por mujeres. La edad fue agrupada en menos de 45 años el 47,5\% (1.323), de 45 a 54 años el $20,9 \%$ (582), de 55 a 64 años el 18,3\% (510) y más de 64 años el $13,2 \%$ (368). El $20 \%$ de la población presentó una puntuación de la escala de riesgo del cuestionario FINDRISC igual o mayor a 15, alto riesgo a muy alto riesgo de padecer diabetes en los próximos 10 años. El 43,38\% presentó un IMC>30 y el 25,97\% declaró recibir medicación para la hipertensión arterial. El 55,37\% refería actividad física baja, el 50,79\% no ingería verduras y frutas en forma diaria y el $17,98 \%$ declaró cifras de glucemias elevadas. Las variables que con mayor frecuencia se asociaron a una escala de riesgo $>15$ fueron: sedentarismo $(80,9 \%)$, cintura $>102 / 88(65,7 / 77,2 \%)$, antecedente de hiperglucemia $(64,0 \%)$, alimentación no saludable $(61,9 \%)$ e IMC>30 (61,8\%). El riesgo $>15$ según IMC fue: IMC <25, el 2,3\%, IMC 25 a 30 el 16,9\%, y el IMC >30 el 45,4\%.

Conclusiones: el $20 \%$ de la población encuestada está en alto riesgo de padecer diabetes. Una de cada dos o tres personas sin diabetes que asisten a un centro de Atención Primaria tiene un FINDRISC $>15$. Esta escala de riesgo es una herramienta simple, económica, de rápida confección, no invasiva y segura para detectar individuos con alto riesgo de padecer diabetes tipo 2. También puede usarse para identificar DM2 no detectada y factores de riesgo de enfermedad cardiovascular.

Palabras clave: diabetes mellitus tipo 2, factores de riesgo, Atención Primaria.

Revista de la Sociedad Argentina de Diabetes 2016; Vol. 50 (96-107)

\section{ABSTRACT}

Objective: to determine the size of the population at risk of developing diabetes mellitus in the population of the municipality of Gral. Pueyrredón who go-attend to Health Centers for Primary Care.

Materials y methods: observational study to determine the risk of developing DM2, through an interview where questions were asked about the 8 questions Questionnaire FINDRISC.

Results: the study sample consisted of 2.784 patients, 54\% were women. Age was grouped in less than 45 years old 47.5 \% (1.323) from 45 to 54 years old 20.9\% (582) from 55 to 64 years old 18.3\% (510), and over 64 years old 13.2\% (368). $20 \%$ of the population has a score of the risk scale questionnaire FINDRISC greater than or equal to 15, high risk to very high risk of developing diabetes in the next 10 years. The $43,38 \%$ showed a BMI $>30$ and $25.97 \%$ was currently receiving medication for high blood pressure. The $55.37 \%$ reported low physical activity, 50.79\% do not eat vegetables and fruits on a daily basis and $17.98 \%$ declared high blood glucose. The variables most commonly associated with a risk score $>15$ were: sedentary lifestyle (80.9\%), waist $>102 / 88$ (65.7/77.2\%), history of hyperglycemia (64.0\%), unhealthy diet (61.9\%) and BMI>30 $(61.8 \%)$. The risk $>15$ according to $B M I$ was: $B M I<25,2.3 \%$, $\mathrm{BMI} 25-3016.9 \%$, and $\mathrm{BMI}>3045.4 \%$.

Conclusions: $20 \%$ of the surveyed population is at high risk for diabetes. One of every 2 or 3 non-diabetic patients attending to a primary care center have a FINDRISC $>15$. This diabetes risk scale is a simple, inexpensive tool, making quick, noninvasive and safe exploration to identify individuals at high risk of developing type 2 diabetes. It also can be used to identify undetected DM2 and risk factors for cardio vascular disease.

Key words: type 2 diabetes mellitus, risk factors, Primary Care.

Revista de la Sociedad Argentina de Diabetes 2016; Vol. 50 (96-107) 
1 Director del Curso de Capacitación en Diabetes para Enfermeros y Equipo Interdisciplinario de Salud (CCD) del Capítulo Atlántico de la Sociedad Argentina de Diabetes (SAD) Región Mar del Plata, Especialista Consultor en Clínica Médica con Certificación Agregada en Diabetes, Director Asociado del HIGA Dr. O Alende Mar del Plata (1983-1988), Jefe del Servicio de Clínica Médica del HIGA Dr. O Alende (20032010), Presidente del Capítulo Atlántico de la SAD (20112012), Miembro Titular de la SAD, Miembro de la Sociedad Latinoamericana de Diabetes, Mar del Plata, Argentina

2 Responsable Médico del Servicio de Endocrinología de la Unidad Asistencial Dr. César Milstein, Directora de la Carrera de Médico Especialista en Endocrinología de la UBA, Médica del Instituto Cardiovascular Buenos Aires, Presidente de la Sociedad Argentina de Diabetes (2015-2016), CABA, Argentina

3 Subdirector del CCD, Especialista Jerarquizado en Clínica Médica, Ex Jefe del Servicio de Clínica Médica del HIGA Dr. O Alende, Prof. Titular de la Cátedra de Nutrición de la Facultad de Ciencias de la Salud de la Universidad de Mar del Plata, Miembro Titular de la SAD, Vicepresidente del Capítulo Atlántico de la SAD, Mar del Plata, Argentina
4 Especialista en Clínica Médica, Magister en Diabetes (USAL), Educadora Certificada en Diabetes por la $S A D$, Miembro de la SAD, CABA, Argentina

5 Ing.MSc. CONICET, Universidad Nacional de Mar del Plata, Mar del Plata, Argentina

6 Integrante del Grupo Interdisciplinario de Salud de la Unidad de Diabetes del CEMA, CABA, Argentina

7 Docente del CCD, CABA, Argentina

8 Lic. en Enfermería, Especialista en Docencia Universitaria, Prof. Adjunta de la asignatura "Enfermería de alto riesgo" de la Lic. en Enfermería de la Universidad de Mar del Plata, Mar del Plata, Argentina

9 Especialista Jerarquizado en Clínica Médica y Endocrinología con Certificación Agregada en Diabetes, Jefe del Servicio de Clínica Médica del Hospital Municipal Sub Zonal Felipe Fosatti de Balcarce (1998-2013), Miembro de la Sociedad Argentina de Endocrinología y Nutrición, Miembro Titular de la SAD, ex Presidente del Capítulo Atlántico de la SAD (20022003), CABA, Argentina

Contacto del autor: Segundo Guzmán Rodríguez

E-mail: segundoguzmanrodriguez@yahoo.com.ar

Correspondencia: Buenos Aires No 3924 (CP7600) Mar del Plata, Prov. de Bs. As. Argentina

Tel.: (011) 0223-4931734

Fecha de trabajo recibido: 01/11/16

Fecha de trabajo aceptado: 12/12/16

Conflictos de intereses: los autores declaran no presentar conflictos de interés

\section{INTRODUCCIÓN}

La diabetes mellitus tipo 2 (DM2) es una de las patologías crónicas no transmisibles con mayor relevancia socio-sanitaria dado que su prevalencia está progresando en forma alarmante.

Los cálculos de la carga mundial de diabetes procedentes de la "Actualización del Atlas de la Diabetes de la Federación Internacional de Diabetes" (FID) para 2014 se publicaron el Día Mundial de la Diabetes (14 de noviembre). Según dichos cálculos, 387 millones de adultos en el mundo viven hoy con diabetes tipo 1 ó 2 y casi la mitad de ellos no lo sabe. Cada año, el número de casos está en aumento, la FID calcula aproximadamente que 592 millones de personas vivirán con diabetes en el año 2035?.

En la región de Sudamérica y El Caribe (SACA) aproximadamente 25 millones de personas tienen diabetes. Si no actuamos ahora, la cifra alcanzará los 38 millones en el año 2035. El 27\% no habrá sido diagnosticado y tendrá un mayor riesgo de desarrollar complicaciones serias y costosas?.

La prevalencia en Argentina -según la Encuesta Nacional de Factores de Riesgo para Enfermeda- des No Transmisibles- es del 9,8\%. Esto significa algo más de 3 millones de personas con diabetes mayores de 20 años. A esto se le deberían sumar las personas en riesgo de padecerla que representan una cifra similar ${ }^{2}$.

Se estima que, en 2014, la diabetes causó unas 219 mil muertes; de ellas, el 45\% habría afectado a menores de 45 años de edad.

El gasto del tratamiento en la región SACA alcanzará los 29 mil millones de dólares, cifra que representa sólo el $5 \%$ del total mundial'. En Estados Unidos el costo del tratamiento de la diabetes se incrementó un 41\% entre 2007 y 2012. Además de esta carga económica, la diabetes implica un incalculable costo social en términos de reducción de la calidad de vida, dolor y sufrimiento de la persona con diabetes, sus familiares y amigos ${ }^{6}$.

Esta realidad requiere implementar en cada región estrategias preventivas para combatir la creciente prevalencia de esta grave y costosa enfermedad ${ }^{5-8}$.

Todo ello ha motivado el desarrollo de diferentes estrategias de cribado para detectar una enfermedad en individuos sin signos o síntomas 
de la misma. La intención del cribado o screening es identificar enfermedades de manera temprana dentro de una comunidad. Esto permite la rápida gestión e intervención con la esperanza que se reduzcan los efectos provocados por la enfermedad.

Los ejemplos más conocidos de escalas de identificación de sujetos con DM no diagnosticada o con riesgo de desarrollar DM2 en los próximos 10 años, basadas en información clínica, son las desarrolladas por la Asociación Americana de Diabetes $^{9}$, Universidad de Maryland, Sociedad Alemana de Diabetes y la Sociedad de Diabetes de Finlandia (cuestionario Finnish Diabetes Risk Score, FINDRISC, Anexo 3).

Todas ellas tienen en común la sencillez en la toma de variables. Presentan similar exactitud diagnóstica, con áreas bajo la curva superponibles a las que añaden variables de laboratorio y superan habitualmente a las que utilizan exclusivamente la glucemia de ayunas ${ }^{3}$. Cualquiera de ellas ofrece similar resultado en la sensibilidad que la sobrecarga oral de glucosa, pero una peor especificidad ${ }^{3}$.

El cuestionario FINDRISC ha demostrado una sensibilidad del $81 \%$ y una especificidad del $76 \%$ para predecir el desarrollo de diabetes mediante la utilización de variables clínicas no invasivas ${ }^{4}$.

Conocer la prevalencia de los estadios tempranos de esta enfermedad en la población requiere implementar estrategias preventivas para combatir la creciente pandemia mundial de esta grave y costosa enfermedad.

Estas estrategias son ampliamente conocidas; se realizaron en pacientes con glucemias de ayunas alteradas en los que se observó el efecto de distintas intervenciones. En los estudios de Malmö ${ }^{10}$, Da Quing ${ }^{11}$ y Finnish DPS ${ }^{12}$ se estudió el efecto de la dieta y la actividad física realizando un seguimiento de seis años, seis años y tres años respectivamente. En ellos se detectó una reducción del riesgo relativo (RRR) de padecer diabetes de un 63,39 y $58 \%$ respectivamente.

En otros estudios como Diabetes Prevention Program (DPP) ${ }^{13}$, XENical in the Prevention of Diabetes in Obese Subjects (XENDOS) ${ }^{16}$, Diabetes Reduction Assessment with Ramipril and Rosiglitazone Medication (DREAM Study) ${ }^{17}$, An International Study on the Efficacy of an $\alpha$-glucosidase inhibitor to prevent type 2 diabetes in a population with impaired glucose tolerance, (STOP-NIDDM) ${ }^{14}$ y Pioglitazone for diabetes prevention in impaired glucose tolerance (ACT NOW Study) ${ }^{15}$, las inter- venciones se referían a distintos agentes terapéuticos (metformina, orlistat, ramipril, acarbose y glitazonas) con seguimientos entre 2,5 a cuatro años. En estos estudios los resultados fueron muy promisorios observándose una RRR que oscilaba entre 23 y $72 \%$, demostrando una muy aceptable efectividad a costos en general extremadamente elevados y con una implementación a gran escala que resulta de muy dificultosa realización en países con bajos recursos.

Si bien es mundialmente reconocido que la lucha contra la enorme carga de la DM2 implicará una serie de múltiples estrategias a este nivel, aquellas que se centran en las iniciativas de prevención primaria -es decir, prevención y detección temprana- han demostrado ser costo-efectivas.

Para implementar estos programas de prevención "en el mundo real" es necesario en primer término conocer la magnitud del problema de cada población.

\section{Identificación de quiénes pueden estar en alto riesgo}

De los distintos protocolos destinados a la detección temprana de la DM2 elegimos la Encuesta FINDRISC ampliamente conocida y validada en estudios previos ${ }^{4}$. Esta encuesta categorizó distintos factores de riesgo asignándole una puntuación a cada una de ellos según su característica. Se seleccionaron factores de riesgo simples que podrían utilizarse convenientemente en la Atención Primaria o aún ser autoadministrados. Se tomaron sólo las variables que eran fáciles de evaluar sin pruebas de laboratorio o aquellas mediciones clínicas que no requirieran habilidades especiales.

La puntuación de esta escala de riesgo es un cuestionario de una página que contiene ocho preguntas, con respuestas categorizadas que incluyen: edad, índice de masa corporal (IMC), circunferencia de cintura, actividad física, consumo diario de frutas o verduras, antecedentes de tratamiento antihipertensivo, nivel elevado de glucemia y familiares de primer y segundo grado con diabetes.

La suma del valor de cada una de las respuestas indica el nivel de riesgo del encuestado. La suma de los ocho ítems dan valores que van de 0 a 26 puntos (Anexo 3).

EI FINDRISC fue desarrollado originalmente en un estudio prospectivo para identificar a las personas en alto riesgo de desarrollar $\mathrm{DM}^{4}$.

En otro estudio se analizó el desempeño de 
esta puntuación como herramienta de cribado para la detección de DM2 no diagnosticada previamente $(11,6 \%$ en hombres y $6,4 \%$ en mujeres), tolerancia a la glucosa alterada $(50,6 \%$ en hombres y $33,3 \%$ en mujeres) y síndrome metabólico demostrando estar estrechamente asociados a ellas y a diversos factores de riesgo de enfermedades cardiovasculares $(E C V)^{19}$.

EI FINDRISC también identifica a las personas que están en alto riesgo (puntuación de 15 a 20) o muy alto riesgo (puntuación $>20$ ) de padecer DM2 y que tienen valores glucémicos normales. Esto permite seleccionar a quienes se les debería poner en práctica la intervención de modificación de su estilo de vida ${ }^{18}$. Esta intervención es la verdadera "prevención primaria de la DM2". La efectividad de esta intervención ha sido demostrada ya en diversos estudios previos, con reducciones del riesgo relativo (RRR) que van desde el 39 al $63 \%$, con seguimientos de 3 a 5 años y un número necesario a tratar (NNT) $=4$ y 10 pacientes año ${ }^{20}$.

En este programa, a la población con puntaje de alto riesgo se le realizó una prueba adicional, como la PTGO, para identificar DM2 sin haber sido detectada previamente; esto es "evaluación individual del alto riesgo". A la población con puntaje inferior se le otorgó información escrita sobre estilo de vida saludable.

Por lo tanto estos procedimientos pueden emplearse tanto como una herramienta para detectar población de alto riesgo de padecer DM2 o para la detección temprana de casos de DM2 y como herramienta de "educación terapéutica" en prevención primaria.

En un país como el nuestro donde la población es tan heterogénea, también la distribución de las enfermedades lo es, por lo que deberían implementarse programas de detección del riesgo y prevención primaria de la diabetes que demostraron ser costo-efectivos en cada región del extenso territorio. Estos programas se ajustarán así a las necesidades y posibilidades de cada una de las personas.

(*) Esquina Saludable es un dispositivo de promoción de la salud mediante la cual se pone a disposición de todos los vecinos de Mar del Plata y Batán la posibilidad de efectuar la valoración del riesgo cardiovascular de forma gratuita en distintos puntos de la Ciudad, durante todo el año. Mar de Plata es la primera ciudad en implementar este Programa coordinado por la Secretaría de Salud de la Municipalidad de Gral. Pueyrredón. Son puestos itinerantes por las esquinas de alto tránsito de los distintos barrios. El procedimiento consta de un cuestionario sobre antecedentes y hábitos, y luego se hace
“La prevención es posible pero debe llevarse adelante en conjunto. El equipo de salud, la comunidad, las entidades de financiamiento, los responsables de las políticas de salud, la industria y los pacientes con diabetes son quienes deben trabajar juntos para lograr una conciencia recíproca" (Gagliardino JJ. "El desafío de la prevención". Jornadas para la prevención de enfermedades crónicas, junio de 2009).

Este trabajo intentará conocer la magnitud del riesgo de padecer diabetes mellitus tipo 2 (DMT2) en la población del Municipio de Gral. Pueyrredón que concurre a los Centros Asistenciales de Atención Primaria. Estos hallazgos se harán llegar tanto a las entidades gubernamentales como a las no gubernamentales a nivel local que deseen sumarse a este llamado mundial de "tratar de desarrollar, identificar y evaluar estrategias rentables de prevención del riesgo de diabetes basadas en los recursos disponibles"7.

\section{MATERIALES Y MÉTODOS}

En el marco del $\|{ }^{\circ}$ y $\|{ }^{\circ}$ Curso de Capacitación en Diabetes para Enfermería y Equipo Interdisciplinario de Salud, dictado por el Capítulo Atlántico de la Sociedad Argentina de Diabetes (región Mar del Plata) conjuntamente con la Unidad de Diabetes del Centro de Especialidades Médicas Ambulatorias de la Secretaría de Salud de la Municipalidad de Gral. Pueyrredón y desarrollado durante los años 2013 y 2014 respectivamente, realizamos un estudio observacional para determinar el riesgo de los habitantes de esta región de padecer DM2.

La población diana incluyó a las personas sin diabetes que acudieron a los distintos Centros de Atención Primaria (CAPs) y a las Esquinas Saludables $\left(^{*}\right.$ ) de Mar del Plata y Batán (Anexos 1 y 2).

La fuente de información fue una entrevista donde se indagaron sobre las ocho preguntas del cuestionario FINDRISC (Anexo 3), con respuestas cortas y cerradas, y puntuaciones predeterminadas que estiman la posibilidad de desarrollar DM2 en los próximos 10 años.

medición antropométrica (peso, talla y circunferencia de cintura), toma de tensión arterial y determinación de glucemia y colesterol capilar.

Allí se establece un riesgo potencial de enfermedad cardiovascular y se da una recomendación personalizada en base a los parámetros evaluados, ofreciendo información de los Centros de Salud y de actividades deportivas gratuitas en los distintos barrios. En cada esquina trabajan profesionales de Enfermería y Nutrición, todos pertenecientes a la Secretaría de Salud. www.mardelplata.gob.ar/Contenido/esquinas-saludables-0. 
Se registraron datos de parámetros antropométricos clásicos bajo normativas unificadas, universalmente aceptadas y publicadas por la $\mathrm{OMS}^{36}$.

Para el registro del perímetro de cintura se utilizó el siguiente instructivo: 1) colóquese a un lado del participante, localice el punto inferior de la última costilla y la cresta ilíaca (parte más alta del hueso de la cadera) y ponga unas marcas con bolígrafo fino; 2) con una cinta métrica, encuentre el punto central entre esas dos marcas e indíquelo; 3) coloque la cinta sobre el punto indicado en la etapa anterior y pídale al participante que se enrolle en ella, verifique que la cinta se encuentre en posición horizontal alrededor de todo el cuerpo del participante; 4) pídale al participante que esté de pie con los pies juntos, que coloque los brazos a cada lado de su cuerpo con la palma de la mano hacia el interior, y que espire despacio; 5) mida el perímetro de cintura y lea la medición.

Los asistentes al " $\|{ }^{\circ}$ y $\| I^{\circ}$ Curso de Capacitación en Diabetes para Enfermeros y Equipo Interdisciplinario de Salud", como actividad práctica en terreno realizaban 10 encuestas o más por encuentro en sus respectivos puestos de trabajo, (12 CAPs, dos hospitales, cinco clínicas, 39 Esquinas Saludables y Batán).

Los encuentros presenciales fueron siete, un sábado por mes de 9 a 16 hs para facilitar las obligaciones laborales de los educandos. El número de personas encuestadas (muestra) fue de 2.784. Los criterios de inclusión fueron todas aquellas personas que asistieron a los Centros de Atención Primaria de Salud mayores de 15 años de edad.

\section{Análisis estadístico}

En primer lugar se realizó la validación de los datos mediante el cálculo automático del índice FINDRISC. Se efectuó la estadística descriptiva de la totalidad de las variables expresadas como media y varianza; para ello se utilizó el paquete estadístico SPSS ${ }^{\circledR}$ y las funciones estadísticas del programa EXCEL ${ }^{\circledR}$. Se separaron los datos por sexo (mujeres/ varones) y se realizaron los cálculos y gráficos correspondientes a los promedios del índice en cinco categorías (menor de 7, 7-11, 12-14, 15-20, mayor a 20). Para las edades e índice de masa corporal se realizaron cálculos de frecuencias relativas.

\section{RESULTADOS}

La muestra del estudio estuvo formada por 2.784 personas. El $54 \%$ de sexo femenino. La edad fue agrupada en menos de 45 años el 47,5\% (1.323), de 45 a 54 años el 20,9\% (582), de 55 a 64 años el 18,3\% (510), y más de 64 años el 13,2\% (368).

Luego de haber procesado y depurado 2.784 cuestionarios de la encuesta FINDRISC y discriminarlos según la escala de riesgo, detectamos que en la población de Gral. Pueyrredón el 29\% se encuentra en muy bajo riesgo de padecer DM2 en los próximos 10 años, menos de 7 puntos, con un riesgo del 1\% (una de cada 100 personas). El $34 \%$ mostró 7 a 11 puntos, un riesgo bajo del 4\% (una de cada 25 personas), un $17 \%$ estuvo entre 12 y 14 puntos, con un riesgo moderado del $17 \%$ (una de cada seis personas), otro $17 \%$ tuvo entre 15 y 20 puntos, alto riesgo, del 33\% de padecer diabetes (una de cada tres personas) y el $3 \%$ con más de 20 puntos, muy alto riesgo, del 50\% (una de cada dos personas) (Figura 1).

La escala de riesgo discriminada por grupo etario se detalla en la Figura 1a. Como es de esperar en la DM2 el grado de asociación con una escala >a 15 se intensifica con el crecimiento de la población. El $83,39 \%$ de las personas con una escala de riesgo $>15$ era mayor de 55 años.

Analizando los datos por sexo se observó casi una idéntica frecuencia en la escala de riesgo en ambos grupos y a su vez los mismos coincidieron con los valores observados en la escala de riesgo total. Las mujeres presentaron un resultado de FINDRISC igual o mayor de 15 puntos en el $20 \%$ de los casos frente a un $21 \%$ en los hombres.

EI IMC estuvo directamente asociado con el incremento en el riesgo de padecer DM2. Así el grupo con IMC menor a $25(n=782)$ presentó un resultado igual o mayor a 15 puntos en el 2,3\%, en el grupo con IMC entre 25 y $30(n=1.194)$ lo hizo un $16,9 \%$ y en el grupo con IMC mayor de 30 $(n=787)$ el $45,4 \%$, con una clara diferencia de su asociación de riesgo entre sus puntos de corte.

Más de la mitad $(55,37 \%)$ de los encuestados no realizaba 30 minutos diarios de actividad física, cifra que resulta similar a la publicada en la Encuesta Nacional de Factores de Riesgo (ENFR) que fue, para el año 2013, de 55,1\%². En estas personas la puntuación de la escala >a 15 se asoció al sedentarismo en el 80,9\% de los encuestados (Tabla 1).

La circunferencia de la cintura $>102 \mathrm{~cm}$ en hombres y $>88 \mathrm{~cm}$ en mujeres se observó en el $40,25 \%$ de los encuestados. Es la segunda variable en frecuencia de asociación con una escala 
de riesgo $>$ a 15 puntos $(65,7 \%$ sexo masculino $y$ $77,2 \%$ en sexo femenino).

La glucemia elevada tuvo una frecuencia del 17,98\% ubicándose en tercer lugar de frecuencia; en ellos la asociación fue del 64,0\% detectando probablemente casos de DM2 no diagnosticada.

El $50,79 \%$ no realizaba un consumo diario de frutas o verduras. A nivel nacional sólo el 4,8\% de la población consume las cinco porciones de frutas o verduras por día recomendadas por la Organización Mundial de la Salud27.

El 25,97\% de los encuestados declaró estar en tratamiento por hipertensión arterial, de ellos el $59,1 \%$ se asoció a un resultado de FINDRISC >a 15 puntos.

El antecedente de diabetes en familiares de $1^{\circ}$ grado fue de $11,03 \%$, y de $2^{\circ}$ grado de $23,64 \%$. La escala de riesgo asociada a esta variable fue de $54,0 \%$ y $23,4 \%$ respectivamente.

Observando la distribución del riesgo >a 15 de la escala y discriminando según la zona en que se efectuó la encuesta (Figura 2), los valores encontrados en instituciones privadas (Anexo 2), Centros de Atención Primaria y Esquinas Saludables fueron $22,12,21,83$ y $20,40 \%$ respectivamente.

Los encuestados en instituciones públicas (HIGA Dr. O. Alende, Instituto Rómulo Echeverry y Centro de Especialidades Médicas Ambulatorio) y Batán fue menor, 18,69 y $18,51 \%$ respectivamente.

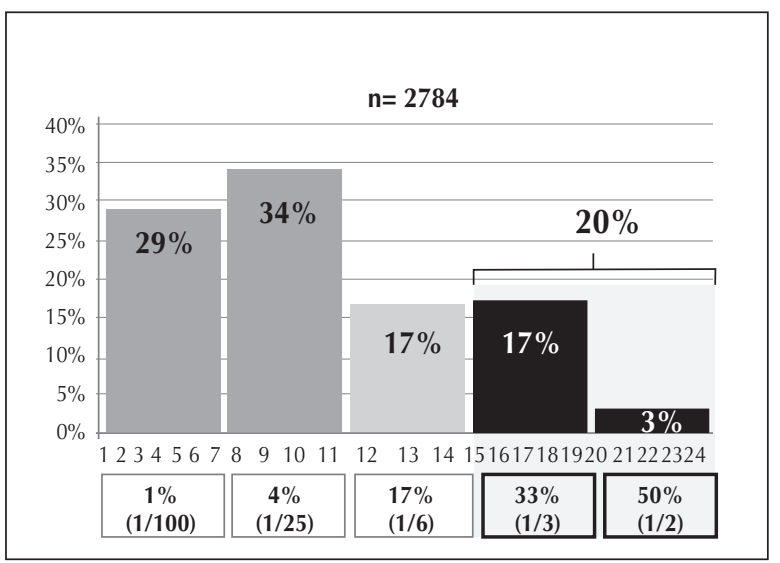

Figura 1: FINDRISC: escala de riesgo total.

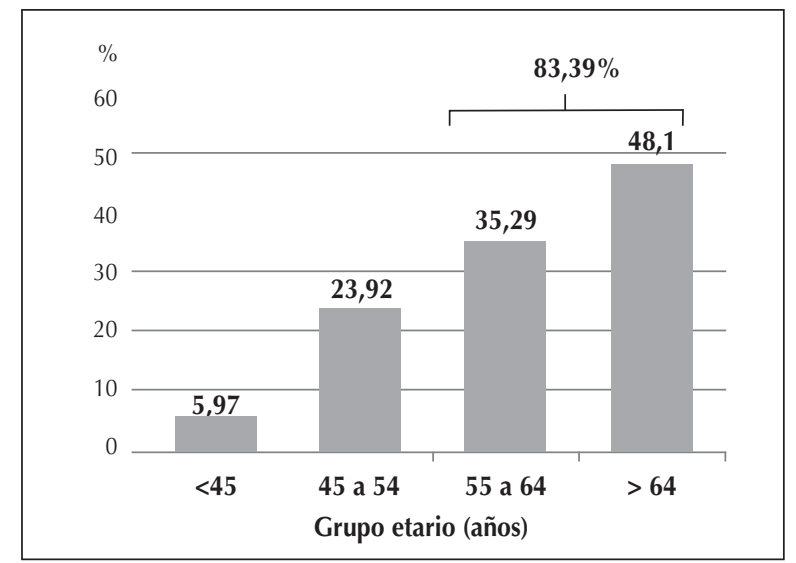

Figura 1a: FINDRISC: escala de riesgo >15 según grupo etario.

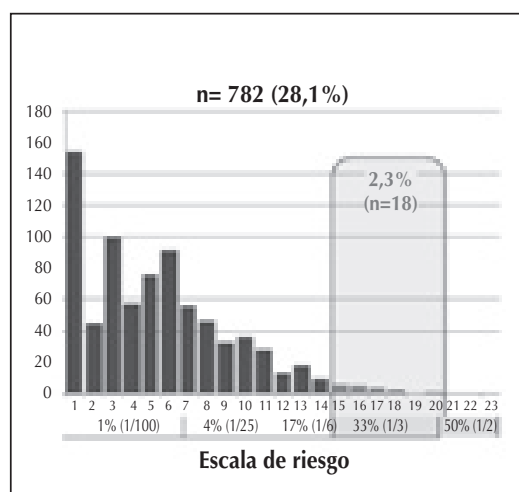

1

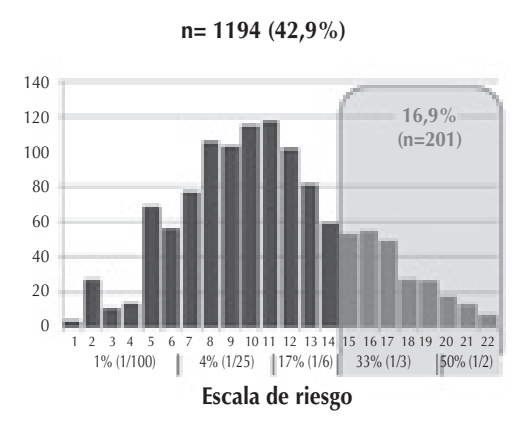

2

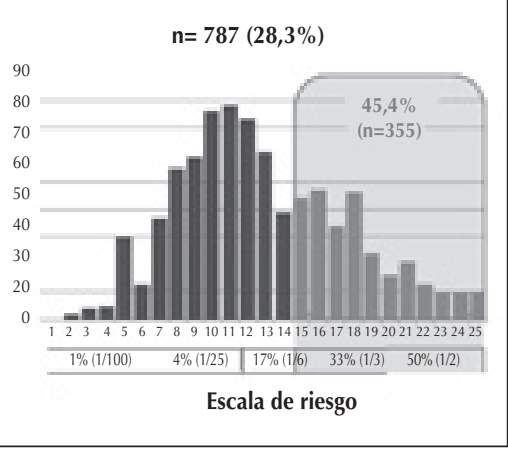

3

Figura 2: 1) FINDRISC: escala de riesgo en IMC $\leq 25 ; 2$ ) FINDRISC: escala de riesgo en IMC de 25 a 30 ; 3) FINDRISC: escala de riesgo en IMC $>30$. 


\begin{tabular}{|c|c|c|c|c|c|c|}
\hline & Masculino & Masculino & Femenino & Femenino & Total & Escala $\geq 15$ \\
\hline Variable & $\mathrm{N}$ & $\%$ & $\mathrm{~N}$ & $\%$ & $\%$ & $\%$ \\
\hline No actividad física & 688 & 53,50 & 848 & 56,23 & 55,37 & 80,9 \\
\hline Cintura $>102 / 88$ & 430 & 33,44 & 687 & 45,56 & 40,25 & $65,7 / 77,2$ \\
\hline Hiperglucemia & 208 & 16,17 & 291 & 19,30 & 17,98 & 64,0 \\
\hline Alimentación & 698 & 54,28 & 711 & 47,15 & 50,79 & 61,9 \\
\hline IMC $>30$ & 391 & 30,04 & 399 & 26,5 & 28,37 & 61,8 \\
\hline HTA & 341 & 22,06 & 381 & 29,8 & 25,97 & 59,1 \\
\hline Familiar de $1^{\circ}$ grado & 280 & 21,77 & 357 & 23,67 & 11,03 & 54,0 \\
\hline IMC 25 a 30 & 554 & 43,08 & 646 & 42,48 & 43,25 & 35,0 \\
\hline Familiar de $2^{\circ}$ grado & 277 & 21,54 & 379 & 25,13 & 23,64 & 23,4 \\
\hline IMC $<25$ & 335 & 26,0 & 449 & 29,77 & 28,16 & 3,1 \\
\hline
\end{tabular}

Tabla 1: Frecuencia de asociación de cada variable con escala FRINDRISC $\geq$ de 15.

\section{DISCUSIÓN}

Los resultados encontrados en este estudio muestran la preocupante realidad de observar que el 20\% de esta población (557 personas) presenta alto riesgo o muy alto riesgo de padecer diabetes, cifra casi idéntica a la obtenida en el estudio de Salinero-Fort en Madrid (19,5\% con puntuación FINDRISC $>15$ puntos).

La frecuencia de obesidad (IMC >a 30) en nuestra población fue del 28,3\%, un 7,5\% superior a la publicada en el año 2013 por la ENFR que fue del 20,8\%, y el sobrepeso (IMC 25 a 30) de 43,2 y $37,1 \%$ respectivamente. A esto se agrega que el $55,37 \%$ de los encuestados no realiza 30 minutos diarios de actividad física, cifra casi idéntica a la de la ENFR que fue del 55,1\%

La frecuencia de HTA encontrada (Tabla 1) fue del 25,97\%, que si bien en nuestro estudio el diagnóstico se efectuó por autorreferencia, es muy parecida a la encontrada en los estudios CARMELA ${ }^{21}$, ( $n=1.482$, dos tomas en una ocasión), realizado en la Ciudad Autónoma de Buenos Aires, que ha comunicado una prevalencia del $29 \%$ entre los 25 y 64 años. En el estudio de Nigro y col. ${ }^{22}$, realizado en la Ciudad de Córdoba en 6.875 personas en quienes se efectuaron también dos registros de HTA, la prevalencia fue del 29,9\%, casi idéntica a la de Deán Funes ${ }^{23}$ con una prevalencia del 29,7\%. Existen estudios de prevalencia de hipertensión entre poblaciones aborígenes como Salta ${ }^{31}$, Chaco ${ }^{32}$, Formosa $^{33}$ y Chubut $^{34}$ donde la frecuencia de hipertensión osciló entre 25,2 y 29,8\%.

La limitación más importante de nuestro trabajo es que la población estudiada no sea representativa del conjunto de los habitantes de esta re- gión, ya que se realizó en personas que asistieron a distintos Centros de Atención Primaria y centros asistenciales en general, y quienes aceptaban voluntariamente conocer su nivel de riesgo de padecer diabetes. Esto puede hacer suponer que la presencia de las distintas variables estudiadas no se corresponda con el valor promedio poblacional y que pudieran presentar mayores comorbilidades por ser personas que asisten por diversos motivos a estos centros asistenciales.

Sin embargo esta muestra investiga el riesgo de diabetes en un amplio sector social, algunos con personas altamente vulnerables como las que asisten a Centros de Atención Primaria periféricos o en Batán en quienes debemos poner más énfasis con medidas preventivas dado que estarán expuestas, por razones de índole socioeconómicas, a una peor situación de riesgo. Es entonces donde mayor importancia tomaría conocer los guarismos de riesgo.

Es ampliamente conocido que los habitantes de medianos y bajos ingresos per cápita a nivel mundial tienen una mortalidad tres veces superior a los de altos ingresos y sólo recibieron el 19,9\% de los recursos destinados a diabetes durante el año 2012. El 77\% de las personas con diabetes vive en países de ingresos medianos y bajos ${ }^{28}$. Una revisión sistemática y metaanálisis donde se revisaron 24 grupos de estudios, con 5.500 participantes ( $43 \%$ masculino), sugiere que las intervenciones en el estilo de vida son efectivas promoviendo la pérdida de peso, la actividad física y dando lugar a una reducción del riesgo de desarrollar diabetes y enfermedad cardiovascular ${ }^{25}$.

Sin embargo, trasladar estas guías de preven- 
ción, con probada evidencia de su efectividad, a la población en riesgo tiene dificultades de implementación. La primera es cómo llegar a la población en riesgo de DM2 en Mar del Plata y zona de influencia.

En el último Censo de 2010 la población de Mar del Plata era de 614.350 habitantes. Según nuestra encuesta se estimaría que 122.870 personas (20\%) están en alto riesgo de padecerla en los próximos 10 años. De ellas, el 61,9\% se presenta en mayores de 55 años; muy pocas reciben intervenciones de estilo de vida y de educación terapéutica. Por lo tanto, existe una urgente necesidad de llegar a grandes números de personas con pre-diabetes con estrategias para generar cambios en su comportamiento a largo plazo.

Como una herramienta más en la intención de llegar con intervenciones efectivas a una gran población, existen protocolos "ongoing" basados en la Web e Internet, como el Alive-PD ${ }^{29}$, que tiene como objetivo desarrollar un programa totalmente automatizado de prevención de la diabetes y determinar su eficacia en un ensayo controlado aleatorio. Este tipo de intervención es una opción más para lograr la factibilidad de llegar a grandes poblaciones con costos razonables. Estas intervenciones de autogestión son necesarias para abordar el control de la diabetes.

Otro estudio probó la efectividad de una intervención conductual telefónica para mejorar el control glucémico en adultos con diabetes. La intervención telefónica entregada por educadores de la salud puede ser una herramienta clínicamente eficaz para mejorar el control de la diabetes.

Programas accesibles y de bajo costo para apoyar la adherencia a la medicación y los hábitos de estilo de vida son fundamentales para mejorar el control glucémico y la obesidad en todas las poblaciones. Una intervención telefónica adaptada a cada región tiene grandes posibilidades de uso a gran escala ${ }^{30}$.

La eficacia de la educación sobre el estilo de vida en la prevención de la DM2 fue ampliamente validada en un metaanálisis ${ }^{37}$ que proporcionó una prueba objetiva de que esta intervención es eficaz para reducir los valores de la tolerancia oral a la glucosa y la incidencia de DM2 en grupos de individuos de alto riesgo.

\section{CONCLUSIONES}

El estudio demuestra que el $20 \%$ de la población encuestada está en alto riesgo de padecer diabetes. En una década dos o tres personas sin diabetes que asisten a un Centro de Atención Primaria tiene un FINDRISC $>15$ quienes seguramente desarrollarán diabetes en los próximos 10 años.

La manera más factible y efectiva de luchar contra esta pandemia sin dudas es la prevención.

En este trabajo, que tiene la virtud de responder a valoración con una escala de riesgo en pacientes que se atendieron por cualquier causa en distintas instituciones de salud de Mar del Plata y alrededores, aporta interesantes datos acerca de cómo en nuestra población adulta la edad, los antecedentes familiares de primer grado de diabetes, la obesidad, el perímetro de cintura aumentado (obesidad central), la HTA y el sedentarismo elevan el riesgo de diabetes 10 veces en los individuos con sobrepeso (IMC 25 a 30) y más de 20 veces si tenían obesidad (IMC >30).

Lo que planteamos aquí es que realizar estas encuestas de escalas de riesgo en poblaciones que consultan en centros de salud por cualquier causa sirve para poner énfasis y alertar a las autoridades sanitarias sobre el alto riesgo de padecer diabetes en el corto plazo que tienen ciertos grupos que, según observamos, podemos identificar claramente y actuar en ellos proponiendo estrategias de cambios de estilo de vida para disminuir ese riesgo.

Los cambios en el estilo de vida producen efectos beneficiosos en los parámetros clínicos y bioquímicos, y disminución del riesgo de diabetes $^{36}$. Este tipo de intervención es una opción viable para prevenir la diabetes tipo 2 y debe implementarse en el Sistema de Atención Primaria de la salud.

\section{Agradecimientos}

Al equipo encuestador: Marcela Adriana Acosta, María Álvarez, Yesica Barraza, Patricia Bazán, Griselda Bozzone, Luciano Cajaravilla, María Florencia Campanella, Vanina Canteros, Patricia Leonor Carrasco, Marcelo Castex, Mercedes Soledad Castro, María Cecilia Corda, Romina D’Ángelo, Héctor Carlos Díaz, Claudia Patricia Díaz, Margarita Díaz, Carina Echinique, Lucía Inés Elissalt, María Ximena Escobar, Yamila Esmal, Norma Galarza, María Cecilia García, María Zulma García, Diego Gardenes, Gabriela Noemí Giménez, Verónica Greco, Diego Martín Laserna, Deborah Maciel, Adriana Elisa Martini, Sofía Orellana, Griselda Emilce Oviedo, Gabriela Pagani, Paula Peláez, Paola Pérez Loyza, María 
Belén Pesce, María Soledad Ramos, Julio Enrique Piconi, María Andrea Ricci, Amalia Roberts, María Rubolino, María Fernanda Santamaría, Carla Romina Simara, Mariana Alejandra Solano, María Elena Soto, Silvia Graciela Stegman, Patricia Tano, Yolanda Nilda Tolosa, Vanesa Vallejos, Claudia Zani.

\section{BIBLIOGRAFÍA}

1. Atlas de la Diabetes de la FID, $6^{\circ}$ Ed. Actualización 2014; Diabetes Voice: Vol. 59, Diciembre de 2014.

2. Tercera Encuesta Nacional de Factores de Riesgo para Enfermedades NoTransmisibles (ENFR). Oct/Dic de 2013.

3. Aekplakorn W, Bunnag P, Woodward M, Sritara P, Cheepudomwit S, Yamwong $S$, et al. A risk score for predicting incident diabetes in the Thai population. Diabetes Care 2006; 29:1872-7.

4. Lindstrom J, Toumilehto J. A practical tool to predict type 2 diabetes risk. Diabetes Care 2003; 26:725-31.

5. Costa B, Barrio F, Bolívar B, Castell C. Prevención primaria de la diabetes tipo 2 en Cataluña mediante la intervención sobre el estilo de vida en personas de alto riesgo. Med Clin (Barc) 2007; 128:699-704.

6. ADA. Economic Costs of Diabetes in the US in 2012. Diabetes Care 2013; Vol. 36, April 2013,1033-1046.

7. Simmons RK, Unwin N, Griffin SJ. International Diabetes Federation: an update of the evidence concerning the prevention of type 2 diabetes. Diabetes Res Clin Pract 2010 Feb; 87(2):143-9.

8. Absetz $\mathrm{P}$, Valve R, Oldemburg B, Heinonen $\mathrm{H}$, et al. Type 2 diabetes prevention in the "Real Word". One-year results of the GOAL Implementation Trial. Diabetes Care 2007; 30:2465-2470.

9. Type 2 Diabetes Risk Test: American Diabetes Association ${ }^{\circledR}$. Disponible en: www.diabetes.org/are-you-at-risk/diabetes-risk-test.

10. Eriksson KF, Lindgärde $\mathrm{F}$. Prevention of type 2 (non-insulindependent) diabetes mellitus by diet and physical exercise. The 6-year Malmö feasibility study. Diabetologia 1991 Dec; 34(12):891-8.

11. Pan XR, Li GW, Hu YH, Wang JX, Yang WY, An ZX, Hu ZX, Lin J, Xiao JZ, Cao HB, Liu PA, Jiang XG, Jiang YY, Wang JP, Zheng $H$, Bennett PH, Howard BV. Effects of diet and exercise in preventing NIDDM in people with impaired glucose tolerance. The Da Qing IGT and Diabetes Study. Diabetes Care 1997; 20: 537-544.

12. Tuomilehto J, Lindström J, Eriksson JG, Valle TT, Hämäläinen $H$, Parikka P, Keinänen-Kiukaanniemi S, Laakso M, Louheranta A, Rastas M, Salminen V, Aunola S, Cepaitis Z, Moltchanov V, Hakumäki M, Mannelin M, Martikkala V, Sundvall J, Uusitupa M. For the Finnish Diabetes Prevention Study Group. Prevention of type 2 diabetes mellitus by changes in lifestyle among subjects with impaired glucose tolerance. N Engl J Med 2001 may; 344:1343-1350.

13. Diabetes Prevention Program Research Group. Reduction in the incidence of type 2 diabetes with lifestyle intervention or metformin. N Engl J Med 2002; Vol. 346, N66.

14. Chiasson JL, Gomis R, Hanefeld M, Josse RG, Karasik A, Laakso $M$, the STOP-NIDDOM Research Group. An international study on the efficacy of an $\alpha$-glucosidase inhibitor to prevent type 2 diabetes in a population with impaired glucose tolerance; rationale, design, and preliminaresceening data. Diabetes Care 1998; 21,1720-1725.

15. De Fonzo RA, Tripathy D, et al. Pioglitazone for diabetes prevention in impaired glucose tolerance (ACT NOW Study). N Engl J Med 2011; 364:1104-15.
16. Torgerson JS, Hauptman J, Boldrin MN, Sjöström L. XENical in the Prevention of Diabetes in Obese Subjects (XENDOS) Study. Diabetes Care 2004; 27:155-161.

17. The DREAM Trial Investigators. Effect of ramipril on the incidence of diabetes. N Engl J Med 2006 oct; 355:1551-1562.

18. Schmiedel K, Mayr A, Fiebler C, Schlager H, Friedland K. Effects of the lifestyle intervention Program GLICEMIA in people at risk for type 2 diabetes: a cluster-randomized controlled trial. Diabetes Care 2015; 38:937-939. DOI: 10.2337/dc14-2206.

19. Saaristo T, Peltonen M, Lindström J, Saarikoski L, Sundvall J, Eriksson JG, Tuomilehto J. Cross-sectional evaluation of the Finnish Diabetes Risk Score: a tool to identify undetected type 2 diabetes, abnormal glucose tolerance and metabolic síndrome. Diabetes Vasc Dis Res 2005; 2:67-72.

20. Alberti KG, et al. Diabetes UK. Diabetic Medicine 2007; 24, 451463.

21. Hernández-Hernández $R$, Silva $H$, Velasco $M$, et al. Hypertension in seven Latin American cities: the Cardiovascular Risk Factor Multiple Evaluation in Latin America (CARMELA) study. J Hypertens 2010; 28:24-34.

22. Nigro D, Vergottini JC, Kushnir E, y col. Epidemiología de la hipertensión arterial en la Ciudad de Córdoba. Rev Fed Arg Cardiol 1999; 28:69-75.

23. Luquez H, Madoery RJ, de Loredo L, y col. Prevalencia de hipertensión arterial y factores de riesgo asociados. Estudio Dean Funes (Provincia de Córdoba, Argentina) Rev Fed Arg Cardiol 1999; 28:93-104.

24. National Cholesterol Education Program. ATP III Guidelines. Disponible en: http://www.nhlbi.nih.gov/files/docs/guidelines/ atglance.pdf.

25. Dunkley AJ, Bodicoat DH, Greaves CJ, Russell C, Yates T, Davies MJ, Khunti K. Diabetes prevention in the real world: effectiveness of pragmatic lifestyle interventions for the prevention of type 2 diabetes and of the impact of adherence to guideline recommendations. A systematic review and meta-analysis. Diabetes Care 2014; 37:922-933. DOI: 10.2337/dc13-2195.

26. Lindström J, Louheranta A, Mannelin M, Rastas $M$, Salminen V, Eriksson J, Uusitupa M, Tuomilehto J. The Finnish Diabetes Prevention Study (DPS): lifestyle intervention and 3-year results on diet and physical activity. Diabetes Care december 2003; 26:3230-3236. Doi:10.2337.

27. Alimentación saludable. Plan Argentina Saludable. Disponible en: www.msal.gov.ar/argentina-saludable/lineas/alimentacion.html.

28. Guariguata L. Diabetes Voice (57) Diciembre 2012.

29. Block G, Azar KMJ, BlockTJ, Romanelli RJ, Carpenter H, Hopkins D, Palaniappan L, Block CH. A fully automated diabetes prevention program, alive-PD: Program Design and Randomized Controlled Trial Protocol. JMIR Res Protoc 2015; 4(1):e3.

30. Chamany S, Walker EA, Schechter CB, Gonzalez JS, Davis NJ, Ortega FM, Carrasco J, Basch CE, Silver LD. Telephone intervention to improve diabetes control: a randomized trial in the New York City. Am J Prev Med 2015 Dec; 49(6):832-41.

31. Coghlan E, Quero B, y col. Prevalencia de hipertensión arterial en una comunidad aborigen del norte argentino. Medicina ( $B$ Aires) 2005; 65:108-112.

32. Bianchi ME, Farias EF, Bolano J, Massari PU. Epidemiology of renal and cardiovascular risk factors in Tobas aborigines. Ren Fail 2006; 28:665-670.

33. Gamarra J, Quintana M, y col. Prevalencia de hipertensión arterial en una comunidad aborigen Qom de la ciudad de Formosa. Revista de Posgrado de la VI Cátedra de Medicina diciembre 2011; N²10:5-6. 
34. Ingaramo RA, Williams D, y col. Prevalence and characteristics of high blood presure in native Americans of Chubut, Argentina. Resumen del 2010 Annual Scientific Meeting and Exposition, American Society of Hypertension, Nueva York, 1 a 4 de mayo de 2010. PO-286.
35. Sección 4: Guía para las mediciones físicas. Disponible en: Step2.www.who.int/chp/steps/Parte3_Seccion4.pdf.

36. Azue K, Oshiro T. Efficacy of lifestyle education to prevent type 2 diabetes. A meta-analysis of randomized controlled trials. Diabetes Care 2005; 28:2780-2786.

\section{ANEXO 1}

\section{Listado de Centros de Atención Primaria de la Salud}

- Subcentro de Salud Estación Camet (CAPS), Ruta 2, Km 393.

- Unidad Sanitaria Chapadmalal (CAPS), 9E/24Y26.

- Unidad Sanitaria Colonia Chapadmalal (CAPS), Ruta 11, Km 34.5, Unidad Turística Chapadmalal.

- Unidad Sanitaria Colonia Barragan Centro (CAPS), Ruta 2, Km 385.

- Unidad Sanitaria El Boquerón (CAPS), Ruta 88, Km 23.

- Unidad Sanitaria Santa Rita (CAPS), Emilio Guanahani 7751.

- Unidad Sanitaria B ${ }^{\circ} 2$ de Abril (CAPS), Soldado Romualdo Pachelzuk 850.

- Unidad Sanitaria 9 de Julio (CAPS), 9 de Julio 7850.

- Unidad Sanitaria F. Ameghino (CAPS), Av. Pedro Luro 10031.

- Unidad Sanitaria Las Américas (CAPS), $202 N^{\circ} 360$ y 12 de Octubre.
- Unidad Sanitaria B ${ }^{\circ}$ Centenario (CAPS), Tierra del Fuego 3116.

- Unidad Sanitaria La Peregrina (CAPS), Ruta 226, Km 16.5.

- Unidad Sanitaria Alto Camet (CAPS), Cura Brochero 3675 y Los Helechos (Ex 182).

- Unidad Sanitaria Las Heras (CAPS), Domingo Heguilor 2750.

- Subcentro de Salud Bo Libertad (CAPS), Onésimo Leguizamón 525.

- Unidad Sanitaria Jorge Newbery (CAPS), Dr. Mariano Moreno 9375.

- Subcentro de Salud Gral. Belgrano (CAPS), 222 y 33.

- Subcentro de Salud El Martillo (CAPS), Génova 6657.

- Departamento de Atención Médica Centro N² (CAPS), Emilio Guanahani 4546.

\section{ANEXO 2 \\ Listado de ubicación de las Esquinas Saludables}

1. Av. Luro e Independencia

2. Av. Paso y San Juan

3. Cerrito y 39

4. Estrada y Tejedor

5. Av. Luro y Av. 180

6. Av. Edison y 12 de Octubre

7. Av. Constitución y Av. 180

8. Av. JP Ramos y Av. F. de la Plaza

9. Funes y Peña

10. Talcahuano y Av. 39

11. Av. Independencia y Quintana

12. Tres Arroyos y Peña

13. Av. Colón y San Juan

14. Av. Juan V Justo 730

15. Expo deporte EMDER MdP

16. Talcahuano y Magallanes

17. Av. Luro y Salta

18. Av. 39 y Juramento

19. Av. Tejedor y Av. Constitución

20. Córdoba y Saavedra
21. Batán

22. Belisario Roldán

23. Hotel NH Provincial

24. Esquina Expo Industria

25. Plaza San Martín

26. Av. Juan B. Justo y San Juan

27. Av. Juan B. Justo y Calle 152

28. Av. Libertad y Arturo Alió

29. Av. Mario Bravo y Vergara

30. Florisbello Acosta y Av. Carlos Tejedor

31. Buenos Aires y Moreno

32. Av. Luro y Dorrego

33. Av. Paso y Urquiza

34. Av. Colón y Mitre

35. Av. Edison y Tripulantes del Furnier

36. San Luis y Brown

37. Av. Juan B Justo y Dorrego

38. Av. Luro y Brasil

39. Av. Jacinto P. Ramos y Génova (CAPS), Emilio Guanahani 4546 


\section{ANEXO 3 \\ Cuestionario FINDRISC}

Cuestionario diseñado por el Dr. Jaako Tuomilehto. Departament of Public Heath, University of Helsinski, and Jaana Lindström, MSF, National Public Health Institute, Finland. Cuestionario publi- cado por The Finnish Diabetes Association, 2001.

Con sólo ocho sencillas preguntas $\mathrm{Ud}$. puede prever cuál es su riesgo de enfermar de diabetes tipo 2 en los próximos 10 años.

\section{Nombre del encuestador:}

\section{¿Qué edad tiene?}

$\begin{array}{ll}\square \text { Menos de } 45 \text { años } & 0 \text { puntos } \\ \square \text { De } 45 \text { a } 54 \text { años } & 2 \text { puntos } \\ \square \text { De } 55 \text { a } 64 \text { años } & 3 \text { puntos } \\ \square \text { Más de } 64 \text { años } & 4 \text { puntos }\end{array}$

¿Ha habido un diagnóstico de diabetes en, por lo menos, un miembro de la familia?

$\square$ No

0 puntos

$\square$ Sí, en mis parientes:

abuelos, tíos, primos

3 puntos

$\square$ Sí, en mi familia directa:

padres, hijos, hermanos

5 puntos

¿Que perímetro de cintura tiene medido a nivel del ombligo?

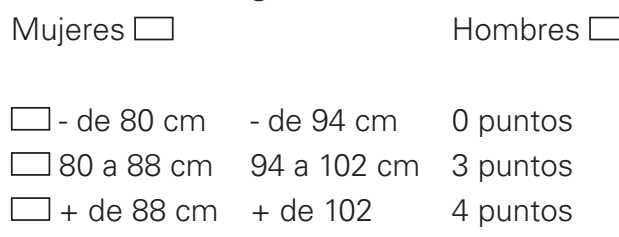

¿Realiza actividad física por lo menos $\mathbf{3 0}$ minutos diarios?

$\begin{array}{ll}\square \text { Sí } & 0 \text { puntos } \\ \square \text { No } & 2 \text { puntos }\end{array}$

¿Con qué frecuencia come frutas, verduras o pan de centeno o integral?

$\begin{array}{ll}\square \text { Diariamente } & 0 \text { puntos } \\ \square \text { No diariamente } & 1 \text { puntos }\end{array}$

Puntaje Total:
¿Le han recetado alguna vez medicamentos para la hipertensión?

$\begin{array}{ll}\square \text { No } & 0 \text { puntos } \\ \square \text { Sí } & 2 \text { puntos }\end{array}$

¿Le han detectado alguna vez un nivel muy alto de glucosa (azúcar) en sangre?

$\begin{array}{ll}\square \text { No } & 0 \text { puntos } \\ \square \text { Sí } & 5 \text { puntos }\end{array}$

¿Cuál es la relación de su estatura y peso (índice de masa corporal)?

$\begin{array}{ll}\square \text { Menos de } 25 & 0 \text { puntos } \\ \square \text { Entre } 25 \text { y } 30 & 1 \text { punto } \\ \square \text { Más de } 30 & 3 \text { puntos }\end{array}$

El índice de masa corporal lo calcula de la siguiente manera: su peso (en $\mathrm{Kg}$ ) dividido por su estatura (en metros) elevado al cuadrado (o simplemente según el cuadro, abajo).

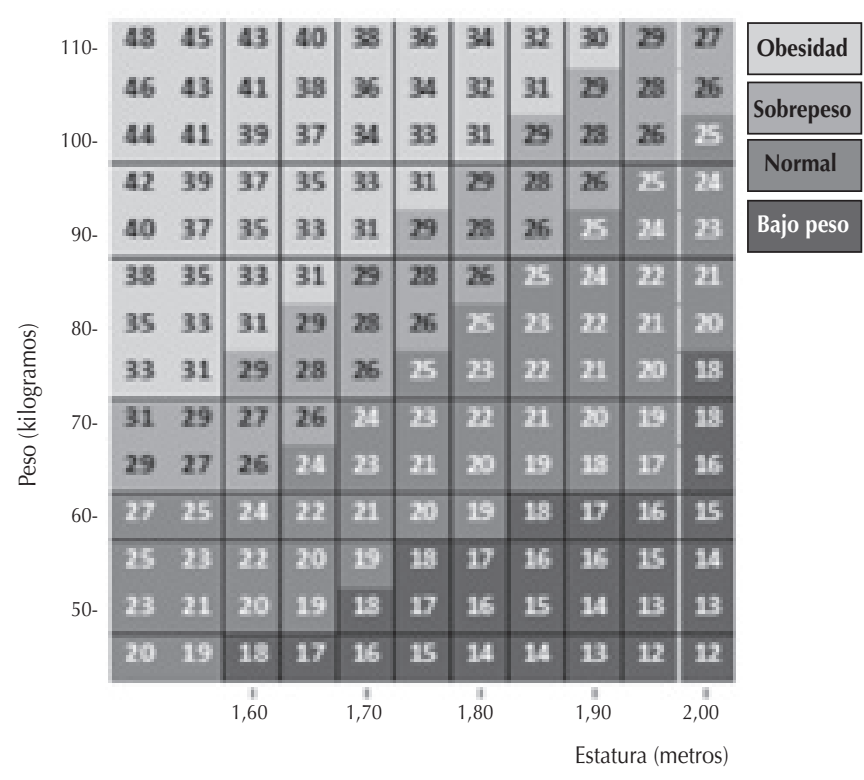


FINDRISC: Escala de riesgo de padecer diabetes tipo (DBT) 2 en 10 años

\section{Menos de 7 puntos. 1 cada 100 personas desarrollarán DBT (1\%)}

Su nivel de riesgo es muy bajo. En su caso no es necesario un cuidado especial o de prevención. Sin embargo no estaría mal cuidar su alimentación y realizar suficiente ejercicio.

\section{De 7 a 11 puntos. 1 cada 25 personas desarrollarán DBT $(4 \%)$}

Para Ud. es recomendable un poco de cuidado, aunque el riesgo de contraer diabetes sea bajo. Si quiere asegurarse siga las reglas:

- En caso de sobrepeso deberá intentar bajar su peso un $7 \%$.

- Manténgase en actividad por lo menos durante media hora, cinco días a la semana.

- La grasa debería constituir, como máximo, sólo un 30\% de su alimentación.

- Consuma diariamente, por lo menos, 30 gramos de fibras vegetales (productos integrales, frutas y verduras)

\section{De 12 a 14 puntos. 1 cada 6 personas desarrollarán DBT (17\%)}

Si Ud. se encuentra en este grupo de riesgo no debería postergar, por ningún motivo, tomar medidas preventivas. En este caso puede recibir consejos e instrucciones de expertos para cambiar su estilo de vida y aplicarlos Ud. mismo. Recurra a un profesional si nota que de esa manera no se puede ayudar.

\section{De 15 a 20 puntos. 1 cada 3 personas desarrollarán DBT (33\%)}

Su nivel de riesgo es muy alto. Subestimar esta situación puede traer graves consecuencias. Lo mejor sería recurrir a ayuda profesional.

\section{Más de 20 puntos. 1 cada 2 personas desarrollarán DBT $(50 \%)$}

Existe la necesidad de actuar inmediatamente, ya que es muy posible que Ud. ya sufra diabetes. Por esta razón debería solicitar una consulta con su médico inmediatamente.

\section{Ud. puede disminuir el riesgo de la siguiente forma \\ Incluso pequeños cambios en su estilo de vida pueden mejorar su salud}

\section{Comer y beber de forma saludable}

Más fruta y verdura

Todos sus alimentos deben contener, en lo posible, mucha fruta y verdura. Lo ideal es que aplaque su hambre solamente con estos alimentos.

Alimentos Al comprar productos lácteos elija pobres en grasa las variantes con poca grasa. Una vez por semana consuma pescado.

Cocinar con Utilice aceite vegetal (o de colza) al poca grasa freír y de oliva en las ensaladas.

Bebidas Evite las bebidas que contienen saludables azúcar.

\section{Más ejercicio en su vida cotidiana}

Tómese tiempo Haga ejercicio 30 a 40 min por día. Elija actividades que pueda acomodar en su vida diaria.

Use el camino al trabajo como Hágalo caminando o en bicicleta. entrenamiento

Pruebe las actividades en grupo

Establecer horas fijas para el deporte y tener compañeros puede ayudar a mantener la motivación en momentos de desánimo.

Los ejercicios correctos

Elija actividades deportivas de intensidad leve a moderada de esfuerzo. La caminata por ejemplo es un deporte ideal.

Si Ud. puede conversar bien durante la práctica del deporte, entonces el esfuerzo que hace es el correcto.

\section{Manténgase activo permanentemente}

Fíjese objetivos Objetivos que no son fáciles de realistas lograr desaniman. Solamente las medidas que son fáciles de cumplir pueden mantenerse en la vida cotidiana.

Fijar los días de Agendar sus días de actividad ayuacción dan a cumplir con sus objetivos.

\section{Entregar al encuestado con la puntuación correspondiente}

Cuestionario diseñado por el Dr. Jaako Tuomilehto. Departament of Public Health, University of Helsinski, and Jaana Lindström, MSF, National Public Health Institute, Finland. Cuestionario publicado por The Finnish Diabetes Association, 2001.

Los autores aclaran: la primera página de la encuesta la retiene el encuestador para su posterior procesamiento. La segunda página se le entrega al encuestado con su puntaje y las recomendaciones que le corresponden (es la primera acción de prevención que se realiza en el momento). 